\title{
A Cross-Layer TCP Modelling Framework for MIMO Wireless Systems
}

\author{
Alberto Lopez Toledo, Student Member, IEEE, Xiaodong Wang, Member, IEEE, and Ben Lu, Member, IEEE
}

\begin{abstract}
We propose a general framework based in the Gilbert model for cross-layer analysis of TCP and UDP over MIMO wireless systems. Our framework takes into consideration diverse system characteristics often difficult to express as a Gilbert model such as fading, space-time transmission schemes, modulation, channel coding and ARQ. We apply our framework to analyze the TCP performance of two representative MIMO systems, namely, the BLAST system and the orthogonal spacetime block coded (STBC) system. In particular, we investigate the optimal information rate that maximizes the TCP throughput, the effect of Doppler on the optimal TCP throughput and the optimal channel coding rate for various modulations. We provide simulations results from the ns-2 network simulator to demonstrate the accuracy of the proposed analytical framework in characterizing the TCP performance. We further apply the framework to two additional cross-layer applications: the analysis of the buffer occupancy on the base station, and the analysis of CBR video transmission over MIMO systems. We show that while the optimal rate for maximum TCP throughput is far from the channel capacity, the optimal rate for error and delay-tolerant video transmission requires much higher rates, and so the physical layer should be aware and adapt to the type of application in order to increase the system performance. We also show that mobility benefits systems with larger buffers, especially for TCP, as the ARQ scheme is able to recover the shorter burst errors. In general, our investigation shows that the type of application plays a crucial role in the optimization of a wireless system, and that our modelling framework is useful for the cross-layer analysis and design of those systems.
\end{abstract}

Index Terms-Cross-layer analysis, MIMO, BLAST, spacetime block coding, information rate, TCP/IP, ARQ.

\section{INTRODUCTION}

$\mathbf{I}$ $\mathrm{N}$ ORDER to seamlessly support a variety of existing and emerging services over wireless networks, it is necessary for the higher layers to exchange information with the physical and the MAC layers, in order to exploit the network resources and to provide optimal inter-operation of applications. The first step towards such a cross-layer approach should encompass an understanding of the variety of the wireless channels and a complete characterization of the upper layers and protocols [14]. Moreover, there is a need for a model that enables the

Manuscript received May 24, 2004; revised March 13, 2005; accepted September 3, 2005. The associate editor coordinating the review of this paper and approving it for publication was X. Shen. This work was supported in part by the U.S. National Science Foundation (NSF) under grant CCR-0207550, and in part by the U.S. Office of Naval Research (ONR) under grant N0001403-1-0039. Alberto Lopez Toledo was supported by Fundación "La Caixa".

A. Lopez Toledo and X. Wang are with the Department of Electrical Engineering, Columbia University, New York, NY 10027 USA (e-mail: \{alberto,wangx\}@ee.columbia.edu).

B. Lu is with Silicon Labs, Broomfield, CO 80021 (e-mail: ben.lu@ silabs.com).

Digital Object Identifier 10.1109/TWC.2006.04026. analytical characterization of the intricate tradeoffs (throughput, time delay, packet loss probability, etc.) that drive the network performance.

While there are several previous work in the cross-layer area ([14]) few research covers completely from the physical to the transport layer (see e.g. [16]). Existing research is either network centered that proposes changes in TCP or in the network layer [13], or physical-layer centered, in which power adaptation and adaptive modulation are taken into account for increasing the physical layer throughput [11]. The application of cross-layer design in the transmission of video over wireless is studied in [10], [16], [18].

In this paper we present a simple framework based on the Gilbert model for the cross-layer analysis of TCP and UDP over wireless systems. While the transition probabilities of the Gilbert model are often difficult to calculate for complex systems, our framework not only provides a method for obtaining these probabilities, but it also takes into account various system parameters at different layers, such as MIMO fading channel characteristic, space-time transmission schemes, modulation, channel coding, ARQ schemes and TCP throughput; enabling the use of well-known existing methods for the analysis of systems based in the Gilbert model for those systems. We apply the proposed modelling framework to cross-layer analysis of MIMO wireless systems employing ARQ for both the BLAST and the orthogonal STBC systems, and study various performance metrics such as the optimal information rate that maximizes the TCP throughput, the effect of Doppler on the optimal TCP throughput, and the optimal channel coding rate that maximizes the TCP throughput under various modulation schemes. We show that the results obtained are very accurate when compared to simulations performed with the ns-2 network simulator, demonstrating the viability of the proposed modelling framework. We further apply the framework to analyze the buffer occupancy at the base station for TCP/ARQ over BLAST and STBC systems, in which the effects of Doppler on the buffer size and the average buffer occupancy at optimal rate are examined. Finally we study the behavior of a CBR video transmission over BLAST and STBC systems, by calculating the maximum throughput of the video system and the optimal coding rate under various modulation schemes.

The remainder of the paper is organized as follows. In Section II, we describe the system under consideration. In Section III we summarize the analytical TCP performance model based on a Gilbert modelling of the physical layer. In Section IV, we show how to obtain the Gilbert model for MIMO wireless links under various scenarios. In Section V, 


$$
\underbrace{\left[\begin{array}{c}
y_{1}(t) \\
y_{2}(t) \\
\vdots \\
y_{n_{R}}(t)
\end{array}\right]}_{\boldsymbol{y}(t)}=\sqrt{\frac{\gamma}{n_{T}}} \underbrace{\left[\begin{array}{cccc}
h_{1,1}(t) & h_{1,2}(t) & \ldots & h_{1, n_{T}}(t) \\
h_{2,1}(t) & h_{2,2}(t) & \ldots & h_{2, n_{T}}(t) \\
\vdots & \vdots & \ddots & \vdots \\
h_{n_{R}, 1}(t) & h_{n_{R}, 2}(t) & \ldots & h_{n_{R}, n_{T}}(t)
\end{array}\right]}_{\boldsymbol{H}(t)} \underbrace{\left[\begin{array}{c}
s_{1}(t) \\
s_{2}(t) \\
\vdots \\
s_{n_{T}}(t)
\end{array}\right]}_{\boldsymbol{s}(t)}+\underbrace{\left[\begin{array}{c}
n_{1}(t) \\
n_{2}(t) \\
\vdots \\
n_{n_{R}}(t)
\end{array}\right]}_{\boldsymbol{n}(t)} .
$$

we validate the framework by comparing it with the ns-2 network simulator, and we present other performance results. In Section VI, we show the capability of the framework to model complex applications; in particular we apply the framework to the buffer occupancy of TCP (Section VI-A) and to the analysis of CBR video transmission over MIMO systems (Section VI-B). Finally, Section VII contains the conclusions.

\section{SYSTEM DESCRIPTIONS}

Physical Layer: Consider a MIMO system consisting of $n_{T}$ transmit and $n_{R}$ receive antennas, signalling through slow Rayleigh fading channels. The input-output relationship of this system is given in (1), where $s_{i}(t) \in \mathcal{A}$ is the transmitted symbol from antenna $i$, with $\mathcal{A}$ being a constellation set with unit energy, i.e., $E\left\{\left|s_{i}(t)\right|^{2}\right\}=1, \boldsymbol{n}(t)$ is ambient noise vector which is circularly symmetric complex Gaussian with independent and identically distributed (i.i.d.) elements, $n_{i}(t) \stackrel{i . i . d}{\sim} \mathcal{N}_{c}(0,1) ; \gamma=\frac{E_{s}}{N_{o}}$ is the signal-to-noise ratio, and $h_{i j}(t)$ denotes the channel gain between the $j$-th transmit and the $i$-th receive antennas at time $t$, and it is a zero-mean complex Gaussian process with the Jakes' correlation model [7].

In this paper, we focus on two MIMO transmission schemes, namely the BLAST method [4], and the orthogonal spacetime block coding (STBC) method [17]. Furthermore, we assume that an ideal channel coding is employed so that we can characterize the physical layer link loss behavior using the maximum information rate for BLAST and STBC systems. Such an assumption is reasonable as capacity-achieving channel codes such as turbo codes and LDPC codes will be employed in next generation wireless systems.

Upper Layers: For the upper layers, a typical TCP/IP/LL/RLP stack is used on the wireless link between the radio network controller (RNC) and the mobile host (MH) (Fig. 1(b)). We do not consider the multiuser scenario in which the medium is shared and a complex MAC protocol is needed, as we are interested only in the performance of end-to-end TCP connections and not in the multiuser interaction. We do not consider other intermediate transport layers such as point-topoint protocol (PPP) as they usually have fixed sizes that generate a constant overhead over the total performance. The RLP layer implements a type of retransmission-repeat request (ARQ) and the link layer performs the fragmentation and reassembly.

Consider the scenario depicted in Fig. 1(a), in which a large data file is transferred through a single TCP connection from a fixed node to a mobile host with guaranteed throughput. For the wired path we assume that no congestion occur and that losses are negligible, as we are only interested in the behavior of TCP in the wireless link. An hybrid ARQ type-I protocol performs retransmissions, fragmentation and reassembly. In ARQ, a frame can be transmitted a number of times until it is successfully received or it is silently discarded when the maximum number of retransmissions is reached (truncated ARQ). In a TCP/RLP system, a TCP frame is divided into $L$ link frames, each of them is transmitted a maximum number of retransmissions $N$. The TCP segment is correctly received when all the $L$ link layer frames are successfully received. In the case of a successful transmission a positive acknowledgement (ACK) is sent back to the transmitter over an errorfree channel and the transmission of a new frame begins immediately. If, on the other hand, the frame is incorrectly received, a negative acknowledgement (NACK) is sent back to the transmitter, which will retransmit the frame in the next time block. If the maximum number of retransmissions is reached, the transmitter discards the frame silently (the upper layer, i.e., TCP, will eventually take care of the error).

\section{TCP/ARQ PERFORMANCE MODEL}

We use a two-state Markov model (i.e. Gilbert model) to model the physical layer transmission success/failure. Specifically, let $S(t)$ be the state of the physical layer link corresponding to the $t$-th frame transmission. Then $S(t) \in\{\mathrm{G}, \mathrm{B}\}$, where $S(t)=\mathrm{G}$ if the $t$-th transmission is successful and $S(t)=\mathrm{B}$ if the $i$-th transmission is erroneous. That is, the physical layer link is modelled by a two-state Markov chain with transition matrix $\boldsymbol{Q}=\left(\begin{array}{cc}1-p & p \\ q & 1-q\end{array}\right)$, where $p \triangleq P[S(t)=\mathrm{B} \mid S(t-1)=\mathrm{G}]$ and $q \triangleq P[S(i)=$ $\mathrm{G} \mid S(i-1)=\mathrm{B}]$. The values $p$ and $q$ summarize the physical layer characteristics such as space-time transmission schemes, MIMO fading channels, modulation and channel coding, etc. We will discuss how to obtain this model in Section IV.

Probability of Packet Loss under ARQ: To calculate the probability of loss of a packet ${ }^{1}$, and the number of frames sent per packet we follow the method in [19]. Given the maximum number of retransmissions $N$ on the link layer and the number of frames per packet $L$, define $\bar{p}_{i}$ as the probability of the $i$-th link layer frame of the current packet being lost in this frame slot given the previous slot state was 'good'. Note that this implies that the $(i-1)$ previous frames were successfully transmitted, since otherwise the packet would have been already discarded. Also define $\bar{q}_{i}$ as the probability

\footnotetext{
${ }^{1}$ Here by packet we refer to an upper layer packet such as IP. Note that the transport protocol encapsulated in the IP packet is not relevant for the probability of loss or the average number of retransmissions, and could be a TCP segment or a UDP packet transporting CBR video.
} 


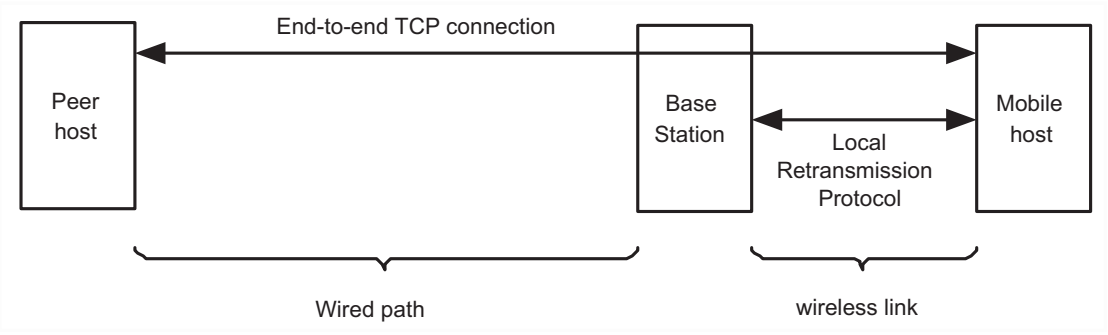

(a) Network scenario.

\begin{tabular}{|c|}
\hline TCP/IP \\
\hline LL \\
\hline RLP/ARQ \\
\hline MAC \\
\hline PHY \\
\hline
\end{tabular}

(b) Stack.

Fig. 1. Network scenario.

of the $i$-th link layer frame corresponding to the current packet being lost in this frame slot, including retransmissions, given that the previous slot state was 'bad'. Note that for the first frame of the packet we have $\bar{p}_{1}$ and $\bar{q}_{1}$ given by

$$
\bar{p}_{1}=p(1-q)^{N-1}, \quad \bar{q}_{1}=(1-q)^{N} .
$$

By induction it is easy to calculate those probabilities for the $L$ frames that constitute a TCP packet as [19]

$$
\begin{array}{r}
\bar{p}_{i}=\bar{p}_{1}+\left(1-\bar{p}_{1}\right) \bar{p}_{i-1}, \quad \bar{q}_{i}=\bar{q}_{1}+\left(1-\bar{q}_{1}\right) \bar{p}_{i-1}, \\
i=2, \ldots, L .
\end{array}
$$

Denote $\pi_{G}$ and $\pi_{B}$ as the steady-state probabilities of $\boldsymbol{Q}$. Then the probability of a packet loss in the current time slot is given by

$$
P_{e}=\pi_{G} \bar{p}_{L}+\pi_{B} \bar{q}_{L}
$$

Average Number of Frames per Packet: We now calculate the average number of frames transmitted per packet, taking into account retransmissions based on [9] and [19]. Note that, when ARQ is in place, a packet consisting of $L$ frames will require more $L$ transmissions to be successfully received if any of the transmissions fails. These retransmissions will affect the delay of the TCP segments, the round trip time and the TCP throughput.

Let $n_{i}$ be the average number of actual frames sent for a packet consisting of $i$ link layer frames given that the first link frame found the channel in a 'good' state; and let $m_{i}^{k}$ be the average number of actual frames sent for a packet consisting of $i$ link layer frames, given that the first frame has already undergone $k$ ARQ retransmissions and the current channel state is (still) 'bad'. Then, the average number of frames sent per packet is given by

$$
F=\pi_{G} n_{L}+\pi_{B} m_{L}^{0} .
$$

In (5), $n_{L}$ and $m_{L}^{0}$ can be calculated recursively as follows [9]: the initialization step

$$
\begin{aligned}
n_{1} & =1 \\
m_{1}^{N-1} & =1 \\
m_{1}^{k} & =1+q n_{1}+(1-q) m_{1}^{k+1}, \quad k=N-2, \ldots, 0
\end{aligned}
$$

and the recursion steps for $i=2, \ldots, L$

$$
\begin{aligned}
n_{i} & =1+(1-p) n_{i-1}+p m_{i-1}^{0}, \\
m_{i}^{N-1} & =1+q n_{i-1}+(1-q) m_{i-1}^{0}, \\
m_{i}^{k} & =1+q n_{i}+(1-q) m_{i}^{k+1}, \quad k=N-2, \ldots, 0 .
\end{aligned}
$$

TCP Throughput: Using the above results, the delay of a TCP packet will be directly proportional to the number of link layer transmissions needed. In the absence of buffer delay, and assuming that the delay of an ACK frame is negligible in the wireless link, the round trip time for a TCP segment is

$$
R T T=2 \cdot T_{f}+F \cdot T_{w},
$$

where $T_{f}$ is the delay of the whole TCP segment through the wired path, $T_{w}$ is the delay of one link layer frame through the wireless interface, and $F$ is the average number of link layer frames sent per TCP segment calculated in (5). Note that $F \geq L$, as $F$ includes the average number of retransmission per frame, and $F \cdot T_{w}$ represents the average delay of the whole TCP segment to be completely transmitted through the wireless link.

We now use the TCP Reno model in [12] to estimate the TCP throughput. In [3] it is shown that in Reno TCP, when the loss rate is low (as in the case of using ARQ), the lossevent rate for TCP, i.e., the fraction of packets that trigger a loss indication at the TCP level, is very close to the TCP packet loss ratio. In that case, equation (4) can be used as an approximation of the loss indication probability ${ }^{2}$. Then, we can approximate the TCP throughput as

$$
\begin{aligned}
& B_{T C P} \approx \min \left(\frac{W_{\max }}{R T T},\right. \\
& \left.\frac{1}{R T T \sqrt{\frac{2 b P_{e}}{3}}+T_{o} \min \left\{1,3 \sqrt{\frac{3 b P_{e}}{8}} P_{e}\left(1-32 P_{e}^{2}\right)\right\}}\right),
\end{aligned}
$$

where $W_{\max }$ is the maximum congestion window size, $b$ is the number of packets acknowledged by a received TCP ACK (usually 2), $T_{o}$ is the initial time-out for the TCP sender, and $R T T$ and $P_{e}$ are the round-trip time and the TCP loss

\footnotetext{
${ }^{2}$ To further support this approximation, we can assume that our system employs some kind of interleaving at the physical layer, making the losses at the TCP level appear as i.i.d., as in [1].
} 
probability calculated in (12) and (4) respectively. We will see in Section $\mathrm{V}$ that this approximation is very good compared with the simulation results.

\section{Calculation of the Gilbert Model parameters FOR MIMO WIRELESS CHANNELS BASED IN MUTUAL INFORMATION.}

While the Gilbert model is extensively used for modelling channels, it is very difficult to calculate the transition probabilities beyond the SISO Rayleigh channel, especially if other system parameters such as coding or antenna diversity are involved. In this section we describe a Monte Carlo method to obtain those Gilbert model parameters for the physical layer of MIMO wireless links. Note that our approach is quite different from the traditional method for single-input single-output Rayleigh fading channel [20], in that here the underlying channel is a MIMO channel and we take into account channel coding.

The basis of our analytical framework is as follows. By the Shannon's channel coding theorem [2], a channel code rate $R$ is achievable if it is such that $R<C$, where $C$ is the capacity of the channel. With the turbo codes and low-density paritycheck (LDPC) codes, we can practically approach Shannon's channel capacity by a fraction of $\mathrm{dB}$ additional transmission power, the small gap which we elect to neglect in this work for simplicity. For the MIMO block-fading channel model in (1), we regard $\mathcal{I}(\boldsymbol{y}(t) ; \boldsymbol{x}(t) \mid \boldsymbol{H}(t))$, the instantaneous mutual information conditioned on the realization of the channel $\boldsymbol{H}(t)$ as 'instantaneous capacity' at time $t$. If the desired transmission rate $R$ is less than the 'instantaneous capacity', error-free transmission is guaranteed (as discussed above); and we claim a 'good' channel state for time $t$,

$$
\mathbf{G} \equiv\{\mathcal{I}(\boldsymbol{y}(t) ; \boldsymbol{x}(t) \mid \boldsymbol{H}(t))>R\} .
$$

Conversely, if the desired rate of transmission $R$ is greater than $\mathcal{I}(\boldsymbol{y}(t) ; \boldsymbol{x}(t) \mid \boldsymbol{H}(t))$, the error-free transmission is not possible (as a matter of fact, the probability of error transmission goes to one); and we claim a 'bad' channel state for time $t$.

In wireless communication systems, the channel response $\boldsymbol{H}(t)$ is typically slowly varying in time, due to Doppler fading. As a result of this, once the MIMO system has moved to a certain state ( $G$ or $B$ ), it may stay over a period of time. We next propose a numerical procedure to capture the dynamic of Doppler fading MIMO channels. More specifically, we generate the instances of $\boldsymbol{H}(t)$ according to the Jakes' model [7], use Monte-Carlo to simulate the state of the system for a long enough period of time, and estimate $p$ and $q$ by simply counting the events of the system going from state $G$ to state $B$ and vice versa.

$$
\begin{aligned}
& \text { for } f_{m} T=\{\text { normalized Doppler range }\} \text { do } \\
& \text { for } R=\{\text { information rate range }\} \text { do } \\
& \text { for } \rho=\{\text { SNR range }\} \text { do } \\
& \text { for } t=1,2, \ldots \text { max Monte-Carlo iterations }\} \text { do } \\
& \quad \text { generate } \boldsymbol{H}(t) \text { according to the Jakes model. } \\
& \text { calculate the instantaneous capacity } \mathcal{I} \text { as in (15)- } \\
& \quad \text { (21). }
\end{aligned}
$$

$$
\begin{aligned}
& \text { if } R<I \text { then } \\
& S(t)=\mathrm{G} \\
& \text { else } \\
& S(t)=\mathrm{B} \\
& \text { end if } \\
& \text { end for } \\
& \text { count the events } G \rightarrow B \text { and } B \rightarrow G \text {, and estimate } \\
& p \text { and } q \\
& \text { end for } \\
& \text { end for } \\
& \text { end for }
\end{aligned}
$$

Unconstrained Constellation Case: For a BLAST system [4] with $n_{T}$ transmit and $n_{R}$ receive antennas and transmitting at a information rate of $R \mathrm{bps} / \mathrm{Hz}$, the instantaneous mutual information conditioned on a particular channel realization $\boldsymbol{H}(t)$ is given by

$$
\begin{array}{r}
\mathcal{I}(\boldsymbol{y}(t) ; \boldsymbol{x}(t) \mid \boldsymbol{H}(t))=\log _{2} \operatorname{det}\left[\boldsymbol{I}_{n_{R}}+\frac{\rho}{n_{T}} R \boldsymbol{H}(t) \boldsymbol{H}^{T}(t)\right], \\
\text { with } \frac{\rho}{n_{T}} R \equiv \frac{\gamma}{n_{T}},
\end{array}
$$

where $\boldsymbol{I}_{n_{R}}$ is a $n_{R} \times n_{R}$ identity matrix, $\boldsymbol{H}(t)$ is the instantaneous channel matrix at time $t$ in $(1) ; \rho=\frac{E_{b}}{N_{o}}$ is the ratio of the average energy per bit with respect to the noise power spectral density, measured at each receiver antenna.

Although suboptimal to BLAST scheme, orthogonal spacetime block codes (STBC) are often considered a low-cost approach to practicing the advantages of MIMO transmission [17]. The mutual information of STBC transmitting at an information rate of $R \mathrm{bps} / \mathrm{Hz}$, is given by

$$
\begin{array}{r}
\mathcal{I}(\boldsymbol{y}(t) ; \boldsymbol{x}(t) \mid \boldsymbol{H}(t))=r_{c} \log _{2}\left[1+\frac{\rho}{n_{T}} R \sum_{j=1}^{n_{T}} \sum_{i=1}^{n_{R}}\left|h_{i, j}(t)\right|^{2}\right], \\
\text { with } \frac{\rho}{n_{T}} R \equiv \frac{\gamma}{n_{T}} .
\end{array}
$$

where $r_{c}$ is the ratio of the number of symbols transmitted to the number of consumed time instances taken, e.g., for Alamouti STBC, $r_{c}=1$; for rate 3/4 STBC, $r_{c}=3 / 4$.

Constrained Constellation Case: So far we have assumed an arbitrary constellation for data symbols; in other words, the information rate $R$ can grow indefinitely with $\rho$. However, in practice, finite MPSK and MQAM constellations are used, so that $R$ is finite and determined by $R=n_{T} M_{c} r$, where $r \leq 1$ is the coding rate of binary error-control codes, $2^{M_{c}}$ is the finiteconstellation size (e.g. $M_{c}=4$ for 16-QAM). For a BLAST MIMO system, the mutual information between the output $\boldsymbol{y}(t)$ and input $\boldsymbol{x}(t)$ with MPSK or MQAM constellations and uniformly distributed symbol probabilities is computed by [6]

$$
\mathcal{I}(\boldsymbol{y} ; \boldsymbol{x})=\mathcal{H}(\boldsymbol{y})-\mathcal{H}(\boldsymbol{y} \mid \boldsymbol{x}),
$$

where $\mathcal{I}$ and $\mathcal{H}$ denote the instantaneous mutual information and entropy function respectively. It is known that $\mathcal{H}(\boldsymbol{y} \mid \boldsymbol{x})=$ $n_{R} \log (\pi e)$ for a Gaussian channel [2]. The entropy of the received signal $\mathcal{H}(\boldsymbol{y})$, however, has to be computed by Monte 


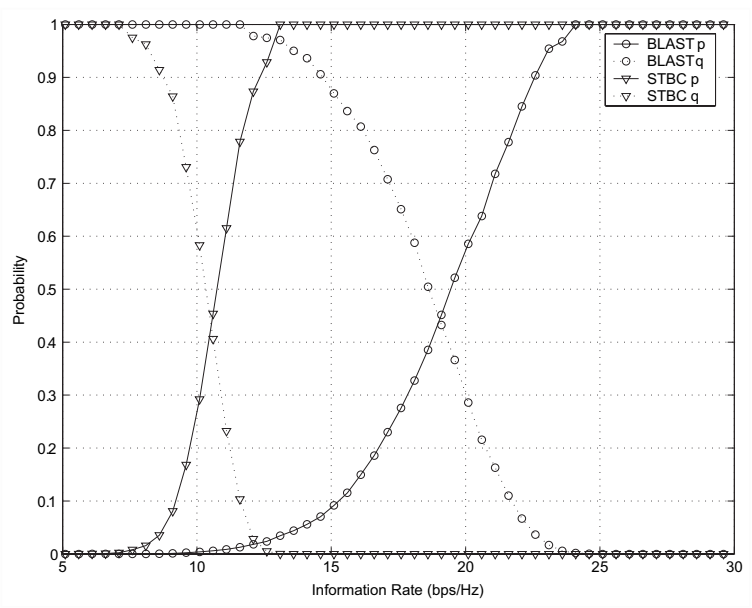

(a) $f_{d} T=0.5$.

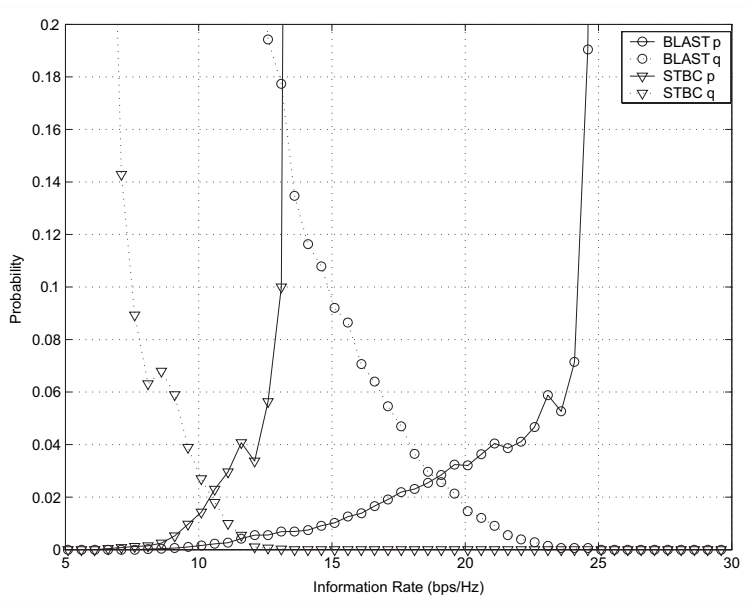

(b) $f_{d} T=0.01$.

Fig. 2. Gilbert model parameters for $2 \times 2$ BLAST and STBC for different Doppler values. $E s / N o=20 \mathrm{~dB}$.

Carlo as follows [6]

$$
\begin{aligned}
& \mathcal{I}(\boldsymbol{y} ; \boldsymbol{x} \mid \boldsymbol{H}) \\
&= \mathcal{H}(\boldsymbol{y} \mid \boldsymbol{H})-\mathcal{H}(\boldsymbol{y} \mid \boldsymbol{x}, \boldsymbol{H}) \\
&= \underbrace{-\mathcal{E}_{n} \log _{2}\left[\frac{1}{2^{n_{T} M_{c}} \pi^{n_{R}}} \sum_{\mathcal{A}^{n_{T}}} \exp \left(-\left\|\boldsymbol{y}-\sqrt{\frac{\rho R}{n_{T}}} \boldsymbol{H} \boldsymbol{x}\right\|^{2}\right)\right]}_{\mathcal{H}(\boldsymbol{y} \mid \boldsymbol{H})} \\
&-\underbrace{n_{R} \log _{2}(\pi e)}_{\mathcal{H}(\boldsymbol{y} \mid \boldsymbol{x})}
\end{aligned}
$$

with $R=n_{T} M_{c} r$, where $\boldsymbol{y}$ is defined in (1); the expectation $\mathcal{E}$ is taken over the random noise $\boldsymbol{n}$ also defined in (1); and the summation $\sum_{\mathcal{A}^{n_{T}}}$ is over all possible values of $\boldsymbol{x} \in \mathcal{A}^{n_{T}}$, in the total amount of $2^{M_{c} n_{T}}$ possibilities. If $M_{c} n_{T}$ is too large the computation in (18) can be approximated using Monte Carlo as follows

$$
\begin{array}{r}
\mathcal{I}(\boldsymbol{y} ; \boldsymbol{x} \mid \boldsymbol{H}) \\
=\underbrace{-\mathcal{E}_{n} \log _{2}\left[\frac { 1 } { \pi ^ { n _ { R } } } \mathcal { E } _ { x } \operatorname { e x p } \left(-\| \boldsymbol{y}-\sqrt{\left.\left.\frac{\rho R}{n_{T}} \boldsymbol{H} \boldsymbol{x} \|^{2}\right)\right]}\right.\right.}_{\mathcal{H}(\boldsymbol{y} \mid \boldsymbol{H})}-\underbrace{n_{R} \log _{2}(\pi e)}_{\mathcal{H}(\boldsymbol{y} \mid \boldsymbol{x})} .
\end{array}
$$

In computing (19), we carry out a large enough number of Monte Carlo runs with two loops: the outer loop generates complex Gaussian noise $\boldsymbol{n}$ according to (1), and the inner loop uniformly generates finite-constellation symbol $\boldsymbol{x}$. The average of all Monte Carlo runs result in an estimate of $\mathcal{I}(\boldsymbol{y} ; \boldsymbol{x} \mid \boldsymbol{H})$.

Since STBC effectively transforms a MIMO channel to a parallel single-antenna (SISO) channels with uncorrelated additive noise and with the same instantaneous channel gain $\sqrt{\frac{\rho R}{n_{T}} \sum_{j=1}^{n_{T}} \sum_{i=1}^{n_{R}}\left|h_{i, j}\right|^{2}}$, the mutual information under con- strained constellation is then computed as:

$$
\begin{gathered}
\mathcal{I}(\boldsymbol{y} ; \boldsymbol{x} \mid \boldsymbol{H})=-r_{c} \mathcal{E}_{n} \\
\log _{2}\left[\frac{1}{2^{M_{c}} \pi} \sum_{\mathcal{A}} \exp \left(-\left|\hat{y}_{1}-\sqrt{\frac{\rho R}{n_{T}} \sum_{j=1}^{n_{T}} \sum_{i=1}^{n_{R}}\left|h_{i, j}\right|^{2}} \cdot x_{1}\right|^{2}\right)\right] \\
-r_{c} \log _{2}(\pi e),
\end{gathered}
$$

with $R=n_{T} M_{c} r_{c} r$; and $\hat{y}_{1}=\sqrt{\frac{\rho R}{n_{T}} \sum_{j=1}^{n_{T}} \sum_{i=1}^{n_{R}}\left|h_{i, j}\right|^{2}} x_{1}+$ $n_{1}$, with $n_{1} \sim \mathcal{N}_{c}(0,1), x_{1} \in \mathcal{A}$, is one of the equivalent SISO channels. If $M_{c} n_{T}$ is too large, (20) can be computed as

$$
\begin{gathered}
\mathcal{I}(\boldsymbol{y} ; \boldsymbol{x} \mid \boldsymbol{H})=-r_{c} \mathcal{E}_{n} \\
\begin{array}{c}
\log _{2}\left[\frac{1}{\pi} \mathcal{E}_{x_{1}} \exp \left(-\left|\hat{y}_{1}-\sqrt{\frac{\rho R}{n_{T}} \sum_{j=1}^{n_{T}} \sum_{i=1}^{n_{R}}\left|h_{i, j}\right|^{2}} \cdot x_{1}\right|^{2}\right)\right] \\
-r_{c} \log _{2}(\pi e) .
\end{array}
\end{gathered}
$$

Note that the definitions of $R$ for constrained-constellation BLAST systems and STBC systems are different, in that an extra multiplier $r_{c}$ is included for STBC; $r_{c}$ reflects the possible transmission inefficiency of STBC, $r_{c}=1$ only if two-transmit-antenna Alamouti STBC is used and otherwise $r_{c}<1$ as shown in [17].

The calculation of the Gilbert model probabilities for other examples of MIMO systems proceed in a similar way, as long as the expression for the instantaneous mutual information is known.

\section{Numerical Results}

In this section we present basic analytical results extracted from the framework, and we validate them with simulations using the ns-2 network simulator.

Gilbert model for MIMO links: Fig. 2(a) shows the values of $p$ and $q$ as a function of the information rate $R$, for $2 \times 2$ BLAST and STBC (rate 3/4) and a normalized Doppler fading 


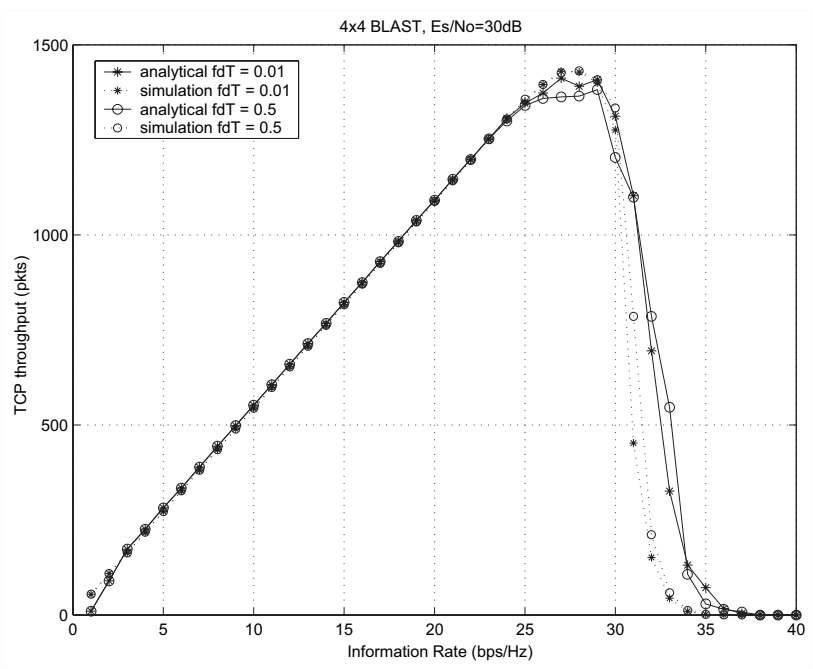

Fig. 3. Comparison of TCP throughput for analytical and simulation results.

$f_{d} T=0.5$ and $E_{s} / N_{o}=20 \mathrm{~dB}$. It is clear that the BLAST system has a larger capacity, as it is able to transmit at a higher information rate without errors, $\sim 12 \mathrm{bps} / \mathrm{Hz}$ versus $\sim 7 \mathrm{bps} / \mathrm{Hz}$ in the STBC case. Also we can see that the STBC system is more reliable, because the parameter $p$ draws a steeper curve than the BLAST case. Interestingly we can see the effect of mobility in Fig. 2(b). Note that the points where the $p$ and $q$ values cross for $f_{d} T=0.01$ is much lower in probability (around $2 \%$, compared to the $50 \%$ in the $f_{d} T=0.5$ case), i.e, for the same SNR the fadings are less frequent but larger for $f_{d} T=0.01$. For TCP with ARQ, this implies that the effect of the mobility is positive: at $f_{d} T=0.01$, although the capacity of the channel does not change compared to $f_{d} T=0.01$, and the probability of going to the 'bad' state $p$ is lower, the probability of getting back to the 'good' state $q$ is only $1 \%$. This implies that when the probability of error blocks starts to increase, the average length of the burst errors is much larger for $f_{d} T=0.01$ than for $f_{d} T=0.5$. This, as we will see, makes the fading correlation (i.e. high mobility) very attractive for FEC and ARQ schemes that will require less retransmissions to approach the capacity curve.

Comparison with ns-2 simulations: Using the previous results we can calculate the TCP throughput for different system configurations. Fig. 3 compares the results obtained for the TCP throughput calculated in (13) and the simulations for a $4 \times 4$ BLAST system with $\frac{E_{s}}{N_{o}}=30 \mathrm{~dB}$. For the simulations we modified RLC module in the GPRS implementation by Richa Jain at IITB (India) to implement a link layer retransmission mechanism for the ns-2 simulator [15]. For the physical layer we generated a sequence of states according to the model in (14). We consider $T$ to be large enough to accommodate a link layer frame, and the transmissions to be synchronized at the beginning of the frame time. For every frame to be transmitted the link controller checks the state of the channel and discards the frame if the state is 'bad', and transmit the frame if the state is 'good'. Fig. 3 shows that our framework is indeed very accurate, as the simulations match almost perfectly the analytical results.

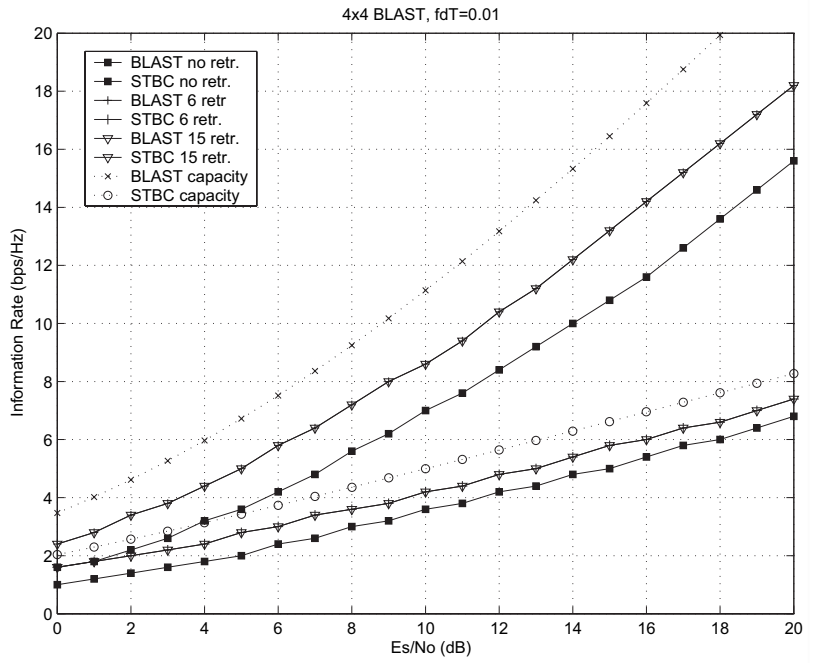

Fig. 4. Information rate that maximizes TCP throughput vs. ARQ persistence.

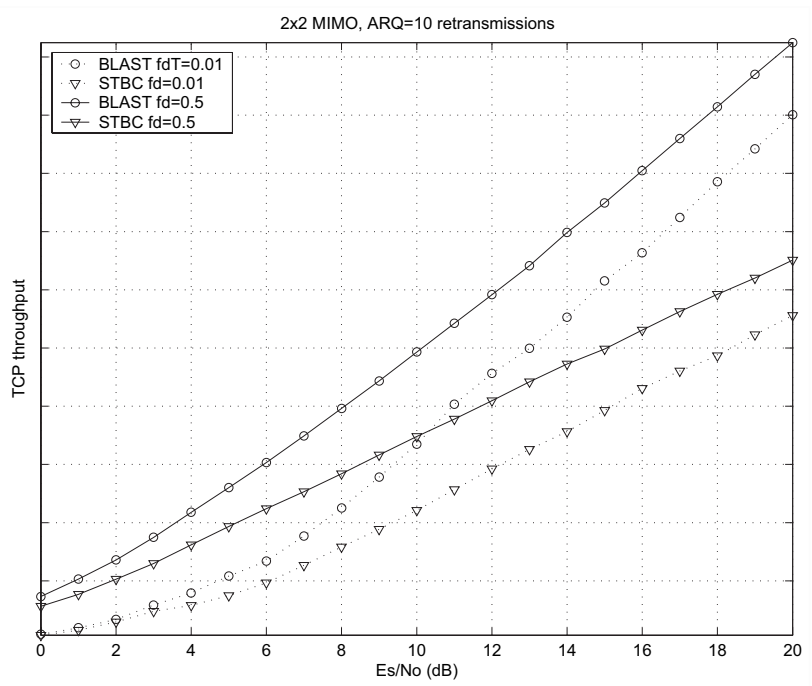

Fig. 5. Comparison of maximum TCP throughput for BLAST and STBC for different Doppler values.

\section{Optimal information rate that maximizes the TCP through-}

put: Using the above method we calculated the optimal rates for all the SNR values. The result, in Fig. 4, shows the information rate that maximizes TCP throughput in a $4 \times 4$ BLAST and STBC (rate 3/4) for $f_{d} T=0.01$. The dashed lines denote the ergodic capacities of BLAST and STBC, and the other lines show the rate at which TCP operates at maximum throughput for different maximum number of retransmissions. It is seen that the optimal rate for TCP is far from capacity irrespective of the number of ARQ retransmission. From a cross-layer design point of view it is an indication that increasing spectral efficiency does not always result in an increment of the TCP throughput. For TCP, it may be preferable a more reliable system because TCP, even when ARQ and combining is used, cannot make use of the additional bit rate. Another comment is that increasing the maximum number of retransmissions over 10 does not provide any benefit to the overall TCP throughput, however it may increase complexity and buffer occupancy, so it may be 


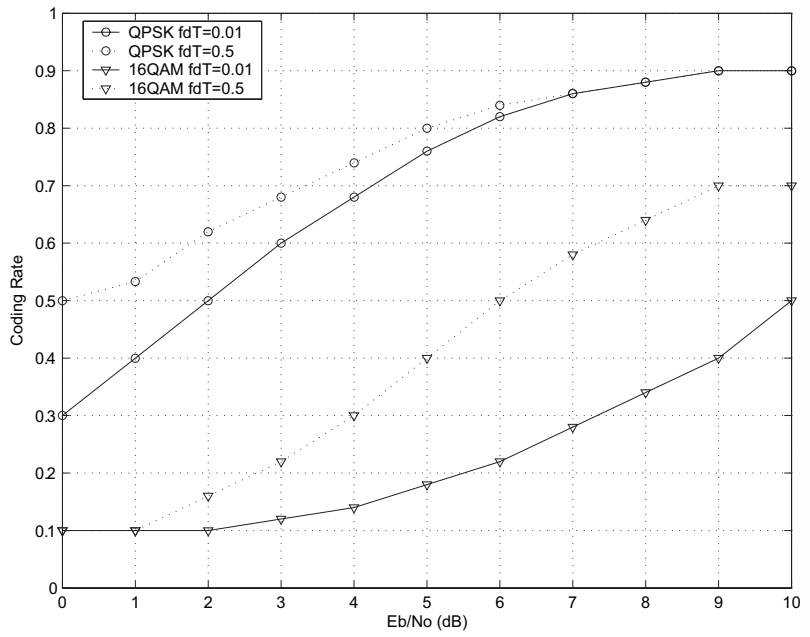

Fig. 6. Optimal coding rate for TCP over $2 \times 2$ BLAST employing QPSK and 16QAM.

interesting to truncate the ARQ persistence.

Effect of Doppler on optimal TCP throughput: Fig. 5 shows the maximum TCP throughput for BLAST and STBC for both $f_{d} T=0.5$ and $f_{d} T=0.01$ and 10 retransmissions. As expected, the values for the TCP throughput are substantially better in the case of high mobility $\left(f_{d} T=0.5\right)$, and only when the SNR reaches $6 \mathrm{~dB}$ the TCP throughput for $f_{d} T=0.01$ begins to grow significatively. It is worth noting that our model captures the effect that mobility has on TCP. While the capacity of a MIMO system does not change with the Doppler, the effects of the time correlation and the deep fadings on TCP are quite significant.

Optimal channel coding rate with finite constellations: In Fig. 6 the optimal coding rates under finite constellations for maximum TCP throughput is showed. We compare the results for QPSK and 16QAM with a maximum ARQ persistence of 10 retransmissions. Note that the QPSK constellation needs less coding than the 16QAM, especially for low SNR. Also it is interesting to note that, for optimal operation, the transmission under $f_{d} T=0.5$ need less coding protection.

\section{Applications of the Framework}

In this Section we show how the model can be applied to the cross-layer analysis of two typical applications in which MIMO systems may be used: buffer occupancy for TCP at the base station and optimal coding rate for a $\mathrm{CBR}$ video source.

\section{A. Analysis of TCP Buffer Occupancy}

Buffer sizing is an important engineering issue as the base station may serve several users, and large buffers create delays and disruption in the traffic patterns. Given the TCP model presented in Section II, if each TCP packet is formed by $L$ link layer frames, then we can assume that the $L$ link layer frames will arrive in bursts with a Bernoulli distribution. We assume that there is a queue at the base station for link layer frames that can accommodate up to $K$ TCP packets, i.e., $L K$ link layer frames. At the beginning of time slot $i$, let $Q_{i}$ denote the

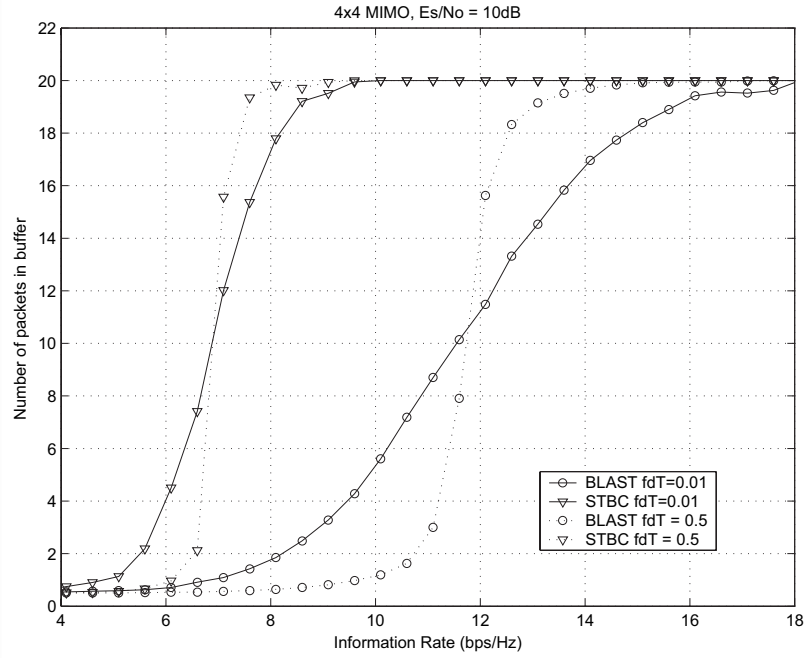

Fig. 7. Comparison of buffer occupancy for $4 \times 4$ BLAST and STBC for different Doppler values. Buffer is 20 packets and $E_{s} / N_{o}=10 \mathrm{~dB}$.

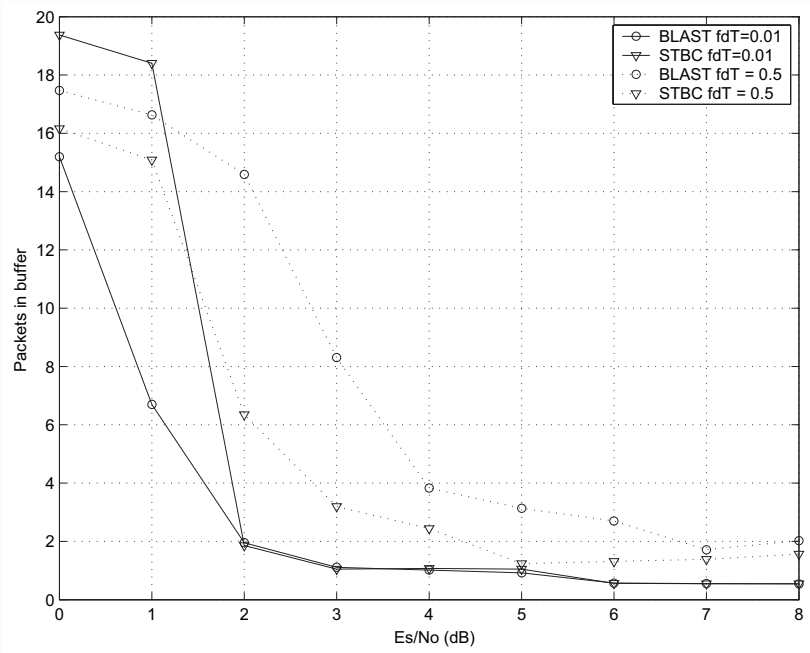

Fig. 8. Average buffer occupancy for $4 \times 4$ BLAST and STBC operating at the optimal rate for TCP for different Doppler values. Buffer is 30 packets.

number of frames in the buffer and $S_{i}$ the state of the channel with $S_{i}=1$ if the channel is 'good' and $S_{i}=0$ if the channel is 'bad'. Then $\left\{\left(Q_{i}, S_{i}\right), i \geq 0\right\}$ is a two-dimension discretetime Markov process. This model has been extensively used in the literature [8], [11].

A TCP packet is lost due to a packet overflow if any of the constituting link layer frames cannot be accommodated in the buffer (i.e., buffer is full). The probability of a TCP packet entering the queue $P_{\text {enter }}$ is

$$
P_{\text {enter }}=\left\{\begin{array}{ll}
0, & Q_{i}>L(K-1) \\
1, & Q_{i} \leq L(K-1)
\end{array},\right.
$$

and the probability of a TCP packet arrival is [8]

$$
P_{a}=B \cdot T \cdot P_{\text {enter }},
$$

where $B$ is the TCP throughput, and $T$ is the time slot corresponding to the duration time of 'good' and 'bad' states of the Gilbert model. The rate at which frames leave the queue is 0 if the channel is in a bad state and 1 if the channel is in 
a good state, meaning that if the channel is bad there can be no departures and when the channel is good, there will be one departure with probability one, if there is at least one frame in the buffer.

With those parameters, the elements of the state transition matrix $\boldsymbol{P}$ can be easily obtained. The stationary distribution of the queue $\left\{\left(Q_{i}, S_{i}\right), i \geq 0\right\}, \pi=$ $\left[\pi_{1,0}, \pi_{1,1}, \ldots, \pi_{1, L K}, \pi_{0,0}, \pi_{0,1}, \ldots, \pi_{0, L K}\right]$, can be obtained by solving $\boldsymbol{\pi}=\boldsymbol{\pi} \boldsymbol{P}$. The average probability of a TCP packet drop in the queue is given by

$$
P_{\text {drop }}=\sum_{Q_{i}=L K-L+1}^{L K}\left(\pi_{0, Q_{i}}+\pi_{1, Q_{i}}\right),
$$

i.e., the probability of finding the buffer with more than $L$ slots free, in which case one of the frames would be dropped and the TCP packet will be lost. The average buffer occupancy is given by

$$
E\{Q\}=\sum_{Q_{i}=0}^{L K} Q_{i} \cdot\left(\pi_{0, Q_{i}}+\pi_{1, Q_{i}}\right) .
$$

The average length of the queue for a TCP packet is [8]

$$
E\left\{Q_{T C P}\right\}=\sum_{Q_{i}=0}^{L K}\left\lceil\frac{Q_{i}}{L}\right\rceil\left(\pi_{0, Q_{i}}+\pi_{1, Q_{i}}\right) .
$$

Applying Little's law we can obtain the average waiting time in the buffer

$$
T_{\text {buffer }}=\frac{E\left\{Q_{T C P}\right\}}{B\left(1-P_{d r o p}\right)},
$$

where again $B$ is the TCP throughput and $P_{\text {drop }}$ is given in (24). Note that the queue itself will have an impact on TCP performance. In particular queuing increases the perceived RTT calculated by TCP, and also it increases the probability of a TCP loss because of the queue limit. Given that, we need to update the expressions for the TCP packet loss probability and RTT as follows

$$
\begin{aligned}
\bar{P}_{e} & =P_{e}+P_{\text {drop }}-P_{e} \cdot P_{\text {drop }} \\
R T T_{q} & =2 \cdot T_{f}+F \cdot T_{w}+T_{\text {buffer }}
\end{aligned}
$$

where $P_{e}$ is the probability of TCP loss including the maximum number of retransmissions (4), $P_{d r o p}$ is the probability of a TCP packet finding the buffer full ${ }^{3}$ (24), $F$ is given by (5) and $T_{b u f f e r}$ is the delay at the buffer. The final expression for the TCP throughput is obtained by substituting the values of (28) and (29) in (13).

Because the queue occupancy depends on the TCP throughput that in turn depends on the queue occupancy itself, a direct calculation of the values is not possible. Here we use an iterative approach: first we calculate the TCP throughput without the queue. The TCP throughput obtained is an upper bound, as the queue will introduce delay. Next, we obtain $T_{\text {buffer }}$ and $P_{\text {drop }}$ and calculate the TCP throughput again. This procedure is repeated until the TCP throughput converges to a fixed value.

\footnotetext{
${ }^{3}$ Note that $P_{e}$ and $P_{d r o p}$ are not disjoint.
}

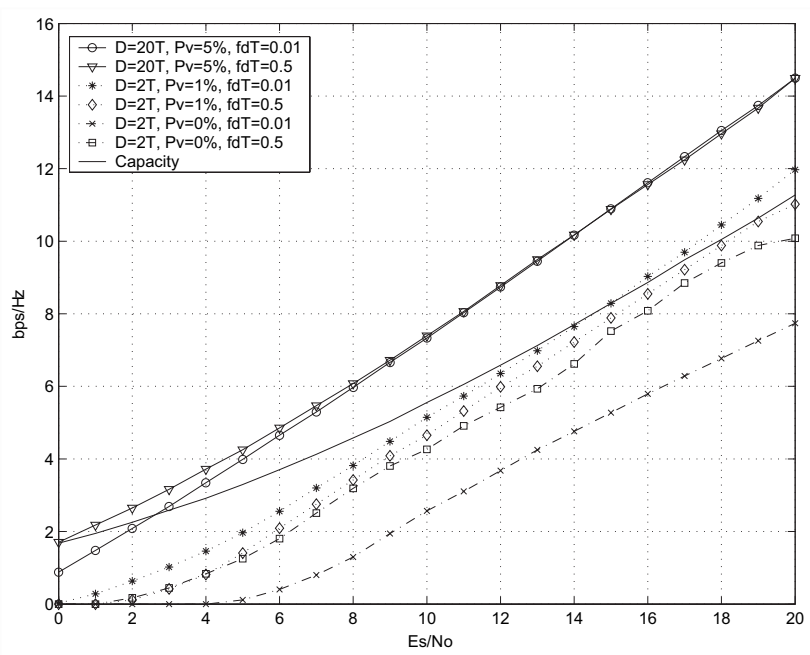

Fig. 9. Rate that maximizes the video throughput for $2 \times 2$ BLAST under different delay and error constraints.

Effect of Doppler on buffer size: Figs. 7 show the behavior of a 20 packets buffer for $4 \times 4$ BLAST and STBC (rate 3/4) systems, at $E_{s} / N_{o}=10 \mathrm{~dB}$ and normalized Doppler fading of $f_{d} T=0.01$ and $f_{d} T=0.5$ respectively. The maximum number of ARQ retransmissions in all cases is 10. It is interesting to see how the vehicle speed has an impact on the buffer size. The highly correlated fadings $\left(f_{d} T=0.01\right)$ have a strong impact on TCP, as it requires more retransmissions, and the queues start to fill quickly. At $f_{d} T=0.5$ the queues start to fill at a higher information rate and more abruptly, usually when the optimal capacity of the channel for TCP is reached. It is also seen that, in general, BLAST has a higher capacity as the buffer saturates at a higher information rate. Also we can see that STBC is more tolerant to the Doppler, as even for $f_{d} T=0.01$ the buffer starts to fill in closer to capacity relative to BLAST.

Average buffer occupancy at optimal rate: Fig. 8 shows the average buffer size at the information rate that maximizes the TCP throughput for BLAST and STBC, with a normalized Doppler fading of $f_{d} T=0.01$ and $f_{d} T=0.5$ respectively. Note that, in general, the buffer requirement for BLAST is higher than for STBC, as the latter is more reliable with shorter burst error lengths. Also, the lower fading correlation at $f_{d} T=$ 0.5 allows the system to reach the maximum TCP throughput with higher average buffer occupancy. This implies that the ARQ system for TCP contributes to increase the maximum TCP throughput due to the shorter (despite more frequent) length of burst errors; and that the systems moving faster have more buffer requirements when operating at the optimal rate.

\section{B. Analysis of CBR Video Transmission}

We can use the proposed modelling framework to analyze the feasibility of MIMO systems for transmitting constant bit rate (CBR) video. The objective is to analyze the behavior of the system for other data sources that do not follow the TCP's AIMD principle, and hence any other kind of crosslayer decision may be needed. We consider CBR video source with a fixed maximum delay $D$ and frame error rate $P_{v}$, where 


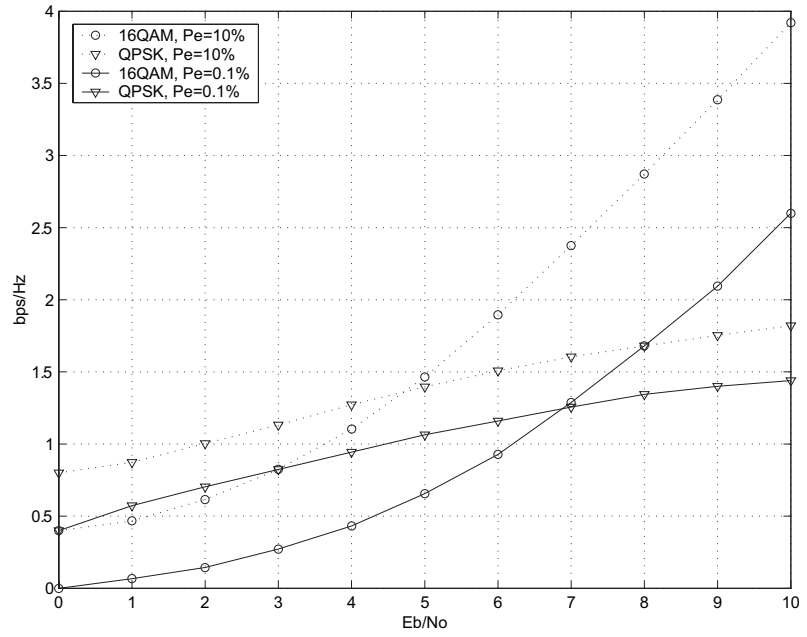

Fig. 10. Video throughput over a $2 \times 2$ BLAST employing QPSK and 16QAM modulations for different frame error targets. Buffer is 20 packets.

the video frames are split into $L$ link layer frames. We also consider a buffered ARQ system on the wireless side similar to the one presented in Section III. Then, the maximum number of retransmissions allowed by the system $N_{\text {video }}$ is calculated by the following condition

$$
T=T_{f}+F_{\text {video }} \cdot\left(T_{w}+\hat{T}_{\text {buffer }}\right) \leq D,
$$

where $F_{\text {video }}$ is the average number of transmissions per frame under the delay constraint, $T_{f}$ is the TCP packet delay in the fixed link, $T_{w}$ is the delay of the frame in the wireless link and $T$ is the packet end-to-end delay. $\hat{T}_{b u f f e r}$ is the buffer delay for each frame, depending on the video rate $B_{v}$, and can be calculated by substituting the values of $B_{v}$ in (23) and (27).

Following the result in (5) for the average number of frames per packet, we can calculate the average number of frames per video packet with the delay constraint considering that

$$
\pi_{G} \hat{n}_{L}+\pi_{B} \hat{m}_{L}^{0}=F_{\text {video }} \leq \frac{D-T_{f}}{T_{w}+\hat{T}_{\text {buffer }}},
$$

where $\hat{n}_{L}$ and $\hat{m}_{L}^{0}$ can be numerically calculated following the scheme in section III. Solving (31) gives the maximum number of retransmissions per video frame $N_{\text {video }}$.

The frame error rate for the video transmission is then obtained by substituting the value of $N_{\text {video }}$ in (6-11) to obtain the average number of frames sent per video frame, and in (28) to calculate the probability of a video packet error $P$. Assuming a maximum tolerable frame error rate for the video of $P_{v}$ (e.g., $1 \%$ for near-unimpaired video quality [5]), we can compare the systems in Sections II for different video codecs, or simply calculate the maximum throughput of a video source to meet the delay and loss requirements.

In order to optimize the system, a direct calculation is not possible and, as in the TCP case, we follow an iterative approach. Starting from the maximum video throughput (line speed), as the quality of the video is the main metric, we first optimize the system to reduce the error rate as much as possible by increasing the number of ARQ retransmissions until the delay constraint is reached or a maximum number of retransmissions is reached. Then, if the error rate is acceptable for the requirement, the system is considered to be in its optimal point; otherwise the video throughput is reduced (and the above calculations are repeated) until it is such that all the requirements are met.

Optimal information rate that maximizes the $C B R$ video transmission: We consider a video transmission over UDP in a scenario similar to the one depicted in Fig.1(a). The base station has a buffer for 20 video packets and ARQ has a maximum of 20 retransmissions. For generality, the delay constraint $D$ is expressed in end-to-end packet transmission delay $T$ (30). We consider three different video applications: a streaming video tolerant to errors in video quality ( $D=20 T, P_{v}=5 \%$ ), for example a web-based video streaming; a real-time video with near-unimpaired video quality $\left(D=2 T, P_{v}=1 \%\right)$, for example a video-conference; and a real-time video with virtually no errors $\left(D=2 T, P_{v} \sim 0 \%\right)$, for example a medical application. Fig. 9 shows the information rate that maximizes the CBR video throughput for the three applications over BLAST (we obtained similar results for STBC), for both $f_{d} T=0.01$ and $f_{d} T=0.5$. The most noticeable result is that the information rate that maximizes the video throughput is greater than the channel capacity for applications that tolerate errors and delay. The streaming application is very tolerant, so transmitting beyond capacity allows the system to operate at optimal throughput considering the transmission errors. Also, the mobility does not significantly affect the performance because the system is allowed a large delay that absorbs all the long fadings with retransmissions, except for low SNRs, in which the $f_{d} T=0.5$ performs better. The video conference application tolerates a $1 \%$ error rate which moves the optimal rate closer to capacity. Finally, the real-time application has the tightest requirements, and so the optimal rate is below capacity in order to maintain the zero error rate. In this case, the low delay requirement prevents ARQ from recovering most of the long burst errors, which makes the $f_{d} T=0.5$ to have better performance.

Video throughput for finite constellations: Fig. 10 shows the video throughput in bps/Hz for 16QAM and QPSK, for $f_{d} T=0.01$ and $f_{d} T=0.5$. In this case we consider a streaming application with $D=20 T$ and a maximum packet error of $10 \%$ and $0.1 \%$ respectively. As shown in the above scenario, the streaming applications with delay tolerance benefit from the mobility of the user. Moreover, in the constrained constellation case it is interesting to see that for low SNR, higher constellations do not provide better throughput, even in the case of high packet error tolerance.

\section{CONCLUSIONS}

We have presented a simple modelling framework for crosslayer analysis of TCP over coded multi-antenna wireless systems. We showed a simple and effective method to calculate the Gilbert model probabilities of a system employing MIMO wireless channels, taking various system parameters into account, including fading channels, space-time transmission schemes (BLAST, STBC), modulation, channel coding and ARQ. The TCP performance predicted by the proposed modelling framework matches well with ns- 2 simulations. We 
showed that the optimal rate at which the TCP performance is maximum is far from the channel capacity, and hence, from a TCP point of view, increasing the channel bandwidth efficiency is not always a good strategy. We also showed that increasing the ARQ retransmissions over certain number does not have a noticeable impact on the TCP throughput. By direct framework application, we showed that the more uncorrelated the channel (higher doppler) the more the TCP/ARQ system can benefit from larger buffers without performance penalty. Finally, we showed that the application requirements affect the optimal rate of transmissions (for error and delay-tolerant video applications, unlike TCP, increasing the transmission rate beyond capacity is a good strategy), and that mobility benefits applications with low delay requirements. As a major point, our investigation shows that the type of application plays a crucial role in the optimization of a wireless system. Parameters such as the delay tolerance or the TCP AIMD feedback scheme drive the system performance, so a crosslayer design approach should take these into account and accommodate the information rate at which the physical layer is transmitting to the real system demands.

\section{ACKNOWLEDGMENT}

The authors would like to thank the editor and the anonymous reviewers for their constructive suggestions and comments during the review process.

\section{REFERENCES}

[1] T. Chahed, A.-F. Canton, and S.-E. Elayoubi, "End-to-end TCP performance in W-CDMA/UMTS," in Proc. IEEE Intern. Conf. Commun. ICC '03, Anchorage, AK, May 2003.

[2] T. M. Cover and J. A. Thomas, Elements of Information Theory. Wiley and Sons, 1991

[3] S. Floyd, M. Handley, J. Padhye, and J. Widmer, "Equation-based congestion control for unicast applications: the extended version," ICSI Tech. Report TR-00-03, Mar. 2000.

[4] G.J. Foschini, "Layered space-time architecture for wireless communication in a fading environment when using multi-element antennas," Bell Labs. Tech. J., 1(2):41-59, 1996.

[5] L. Hanzo, P. Cherriman, and J. Streit, Wireless Video Communications: Second to Third Generation Systems and Beyond. New York, NY: WileyIEEE Press, 2001.

[6] B. M. Hochwald and S. ten Brink, "Achieving near-capacity on a multiple-antenna channel," IEEE Trans. Commun., vol. 51, no. 3, pp. 389-399, Mar. 2003.

[7] W. C. Jakes and D. C. Cox, Microwave Mobile Communications. New York, NY: Wiley-IEEE Press, 1994.

[8] H. Jiang and W. Zhuang, "Quality-of-service provisioning to assured service in the wireless internet," in Proc. IEEE GLOBECOM'03, San Francisco, CA, Dec. 2003.

[9] A. Kumar and J. Holtzman, "Comparative performance analysis of versions of TCP in a local network with a mobile radio link," Sadhana: Proc. in Eng. Sciences, vol. 23, no. 1, pp. 113-129, Feb. 1998.

[10] W. Kumwilaisak, T. Hou, Q. Zhang, W. Zhu, C.-C.J. Kuo, and Y.-Q. Zhang, "A cross-layer quality-of-service mapping architecture for video delivery in wireless networks," IEEE J. Select. Areas Commun., vol. 21, no. 10 , pp. $1685-1698$, Dec. 2003.

[11] Q. Liu, S. Zhou, and G. B. Giannakis, "Cross-layer combining of queuing with adaptive modulation and coding over wireless links," in Proc. MILCOM Conf., Boston, MA, Oct. 2003.

[12] J. Padhye, V. Firoiu, D. F. Towsley, and J. Kurose, "Modeling TCP throughput: a simple model and its empirical validation," IEEE/ACM Trans. Networking, vol. 8, no. 2, pp. 133-145, Apr. 2000.

[13] V. T. Raisinghani, A. K. Singh, and S. Iyer, "Improving TCP performance over mobile wireless environments using cross layer feedback," in Proc. IEEE Int. Conf. Personal, Wireless Commun. (ICPWC), New Delhi, India, Dec. 2002.
[14] S. Shakkottai, T. S. Rappaport, and P. C. Karlsson, "Cross-layer design for wireless networks," IEEE Commun. Mag., vol. 41, no. 10, pp. 74-80, Oct. 2003.

[15] Network simulator 2, http://www.isi.edu/nsnam/ns

[16] P. M. Soni and A. Chockalingam, "Performance analysis of UDP with energy efficient link layer on markov fading channels," IEEE Trans. Wireless Commun., vol. 1, no. 4, pp. 769-780, Oct. 2002.

[17] V. Tarokh, H. Jafarkhani, and A.R. Calderbank, "Space-time block codes from orthogonal designs," IEEE Trans. Inform. Theory, vol. 45, no. 5, pp. 1456-1467, July 1999.

[18] M. van der Schaar, S. Krishnamachari, S. Choi, and X. Xu, "Adaptive cross-layer protection strategies for robust scalable video transmission over 802.11 WLANs," IEEE J. Select. Areas Commun., vol. 21, no. 10, pp. 1752-1763, Dec. 2003.

[19] H. Le Vu and S. Hanly, "A study of TCP performance and buffer occupancy over a fading wireless link," in Proc. IEEE GLOBECOM'01, San Antonio, TX, Nov. 2001.

[20] M. Zorzi, R. R. Rao, and L. B. Milstein, "On the accuracy of a firstorder markov model for data transmission on fading channels," in Proc. IEEE ICUPC'95, Tokyo, Japan, Nov. 1995.

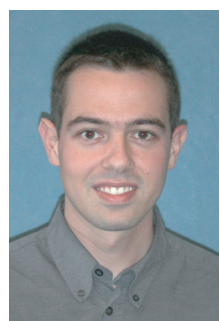

Alberto Lopez Toledo graduated in Computer Engineering (with highest honors) from the University of Murcia (UMU), Spain, in 1999, and obtained the M.Phil. in Computer Science from the same university in 2002. He received the M.Sc. degree in Electrical Engineering from Columbia University, New York, in 2003, where he is currently a Ph.D. candidate in Electrical Engineering. His research interests are in the area of wireless and mobile networking and cross-layer design. He received the Spain's National Academic Excellence Award, the Edwin Howard Armstrong Memorial Award, the La Caixa Foundation fellowship and the Rafael del Pino Foundation fellowship.

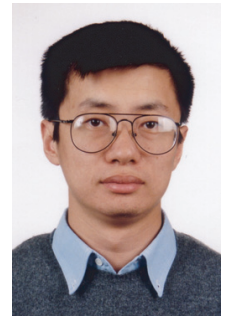

Xiaodong Wang (S'98-M'98-SM'04) received the B.S. degree from Shanghai Jiao Tong University, Shanghai, China; the M.S. degree from Purdue University; and the Ph.D degree from Princeton University, all in Electrical Engineering. From July 1998 to December 2001, he was on the faculty of the Department of Electrical Engineering, Texas A\&M University. Since January 2002, he has been with the Department of Electrical Engineering, Columbia University.

Dr. Wang's research interests fall in the general areas of computing, signal processing and communications, and has published extensively in these areas. Among his publications is a recent book entitled "Wireless Communication Systems: Advanced Techniques for Signal Reception", published by Prentice Hall in 2003. His current research interests include wireless communications, statistical signal processing, and genomic signal processing. Dr. Wang received the 1999 NSF CAREER Award, and the 2001 IEEE Communications Society and Information Theory Society Joint Paper Award. He currently serves as an Associate Editor for the IEEE Transactions on Communications, the IEEE Transactions on Wireless Communications, the IEEE Transactions on Signal Processing, and the IEEE Transactions on Information Theory.

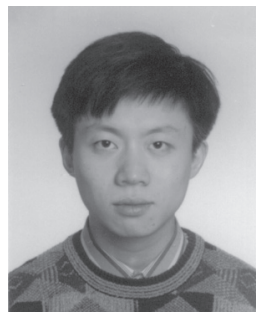

Ben Lu received the B.S. and M.S. degrees in electrical engineering from Southeast University, Nanjing, China, in 1994 and 1997; the Ph.D. degree from Texas A\&M University in 2002.

From 1994 to 1997, he was a Research Assistant with National Mobile Communications Research Laboratory at Southeast University, China. From 1997 to 1998 , he was with the CDMA Research Department of Zhongxing Telecommunication Equipment Co., Shanghai, China. From 2002 to 2004, he worked for the project of high-speed wireless packet data transmission (4G prototype) at NEC Laboratories America, Princeton, New Jersey. He is now with Silicon Laboratories. His research interests include the signal processing and error-control coding for mobile and wireless communication systems. 